\title{
Diferencias transculturales en bienestar subjetivo: México y España
}

\author{
Mónica Carballeira ${ }^{*}$, José-Ángel González² y Rosario J. Marrero ${ }^{1}$
}

${ }^{1}$ Universidad de La Laguna (España)

${ }^{2}$ Universidad de San Luis Potosí (México)

\begin{abstract}
Resumen: El propósito de esta investigación ha consistido en analizar las diferencias en bienestar subjetivo en 346 estudiantes universitarios pertenecientes a dos culturas: colectivista (México) e individualista (España). El bienestar subjetivo global se evaluó mediante la satisfacción vital, la felicidad, las emociones positivas y negativas; considerándose además, cuatro indicadores de satisfacción en dominios específicos: sentimental, estudios, salud y ocio. Los resultados indican que los estudiantes mexicanos puntúan más alto que los españoles en todos los indicadores de bienestar subjetivo. Los análisis de regresión múltiple revelan la importancia de la satisfacción sentimental y con los estudios en la felicidad y satisfacción vital de ambas culturas. Además, son importantes en la predicción del bienestar subjetivo global, la satisfacción con la salud para los mexicanos y la satisfacción con el ocio para los españoles. Los resultados apoyan el efecto diferencial de la cultura en el bienestar, fundamentalmente en su predicción a través de la satisfacción en ciertos dominios específicos.

Palabras clave: Bienestar subjetivo; individualismo; colectivismo; satisfacción vital; emociones; felicidad.
\end{abstract}

\begin{abstract}
Title: Cross-cultural differences in subjective well-being: Mexico and Spain.

Abstract: The objective of this study was to analyse the differences in subjective well-being in 346 undergraduate students, from two cultures: one collectivist (Mexico) and one individualist (Spain). Global Subjective Wellbeing was assessed through the Satisfaction with Life Scale, the Subjective Happiness Scale, the PANAS scales, and also the satisfaction with four specific life domains: affective relations, studies, health and leisure were considered. The analyses have shown higher levels in all the indicators of subjective well-being for Mexican students. The regression analyses revealed the importance of sentimental and studies satisfaction for happiness and life satisfaction in both cultures. Also, health satisfaction for Mexicans, and leisure satisfaction for Spanish influenced global measures of subjective well-being. The findings supported the differential effect of culture on subjective well-being, especially in its prediction through the satisfaction with certain specific life domains.

Key words: Subjective well-being; individualism; collectivism; life satisfaction; emotions, happiness.
\end{abstract}

\section{Introducción}

Uno de los principales enfoques de la Psicología transcultural se ha dirigido al estudio de la clasificación de las sociedades según el predominio del individuo, o bien del grupo; proponiendo el eje "individualismo-colectivismo" (Triandis, 1980); y a analizar las implicaciones que esto tiene en el comportamiento de los individuos. Las culturas individualistas enfatizan la independencia de los individuos mientras que las colectivistas dan prioridad a la interdependencia y las relaciones cercanas (Triandis, 1995). Para las sociedades colectivistas el bien del grupo es más importante que el individual; por lo tanto las normas son básicas. Valores como la seguridad, la obediencia o el conformismo son primordiales en este tipo de sociedades. Por el contrario, las sociedades individualistas están más volcadas en la búsqueda de objetivos y deseos propios, promoviendo la autonomía, a través de actitudes y valores como el logro, la competencia y el placer (Triandis, 1990).

La investigación ha plasmado el mayor interés por las emociones positivas en las culturas individualistas, vinculando una vida de sensaciones placenteras al logro de necesidades y metas, ofreciendo información relevante sobre la satisfacción vital (Schimmack, Radhakrishnan, Oishi, y Ahadi 2002). En las culturas colectivistas, aunque el logro de las metas personales es también importante, sus miembros las subordinan a los intereses de los miembros de su grupo, ya que cumplir con las normas es más importante que maximi-

* Dirección para correspondencia [Correspondence address]

Mónica Carballeira Abella. Universidad de La Laguna. Facultad de Ciencias de la Salud. Sección Psicología. Departamento de Psicología Clínica, Psicobiología y Metodología. Apdo. 456. Campus de Guajara s/n. 38200 La Laguna. Santa Cruz de Tenerife (España). E-mail: mabella@ull.edu.es zar el placer (D’Anello, 2006). Tal y como destacan Furman et al. (2009), el estudio de los valores culturales del colectivismo y su orientación comunal parecen propios de los países latinos y sirven para mitigar la tensión, proporcionando sentido de pertenencia y respeto al individuo. Existen otros grupos culturales como los orientales, los hindúes o grupos indígenas, en los que predominan los valores centrados en la colectividad. Sin embargo, en este trabajo nos interesa analizar la incidencia y los determinantes del bienestar en dos sociedades, a priori similares por su idioma e idiosincrasia -la mexicana y la española- pero que se caracterizan, en el primer caso, por formar grupos cohesivos más fuertes, mientras que en la cultura española, los lazos entre las personas parecen menos intensos, estando más influenciados por los condicionantes de las sociedades individualistas (Hofstede, 1999).

El constructo de bienestar subjetivo estaría compuesto por la satisfacción con la vida, o componente cognitivo; y el balance emocional, o componente afectivo (Diener, Suh, Lucas y Smith, 1999). La satisfacción con la vida representa la discrepancia percibida entre las aspiraciones personales y sus logros, cuyo amplio rango evaluativo va desde la sensación de realización personal hasta la experiencia vital de fracaso o frustración (Veenhoven, 1984). El componente afectivo se relaciona con la presencia de emociones positivas frente a negativas y es lo que se ha denominado felicidad. Las emociones son un reflejo de cómo impactan los eventos vitales en la vida personal; y a partir de todo ello, cada uno de nosotros realiza amplios juicios vitales a nivel global, como un todo; pero también en particular, analizando parcelas específicas como por ejemplo, la del trabajo o la de la pareja (Diener, 2000). De ahí, la relevancia del estudio de ciertos indicadores específicos del bienestar subjetivo, como la satis- 
facción con los dominios vitales citados (Argyle, 1992; DeNeve y Cooper, 1998; Gómez, Villegas, Barrera y Cruz, 2007).

Aunque la mayor parte de las investigaciones que analizan el bienestar en diferentes sociedades han partido de una consideración universal de este constructo, sus determinantes no parecen ser idénticos en grupos culturales distintos. La satisfacción con la vida y los estados emocionales ratifican la importancia de observar diferencias en el comportamiento de las personas provenientes de distintos ecosistemas y con ello, la manera en cómo perciben e interpretan las demandas del grupo social al que pertenecen (Vera, Laborín, Córdova y Parra, 2007). Los juicios sobre la satisfacción vital están determinados tanto por factores psicológicos como por factores culturales (Schimmack et al., 2002), encontrándose diferencias de bienestar personal en la investigación transcultural previa (Diener, 2000; Inglehart, 1990; Rice y Steele 2004; Veenhoven, 2007). Sin embargo, mientras que algunos estudios han hallado que las sociedades individualistas son más felices al obtener logros orientados a metas personales, dando más valor a los sucesos vitales, tanto positivos como negativos, atribuyendo sus acciones a sí mismos, pudiendo dar poca importancia, incluso obviar, aspectos insanos de su entorno social (Triandis, 2005); otros trabajos indican que las sociedades colectivistas muestran mayor bienestar en la medida en que se sienten integradas en un grupo de pertenencia, lo que puede llevar a una estructura social más segura (Díaz y González, 2011).

Algunos estudios que han analizado las causas de las diferencias en bienestar entre diversos grupos culturales, se han centrado en los contrastes a nivel económico, hallándose una asociación positiva entre ingresos económicos y bienestar (Veenhoven, 2007). Diener (2000) informó que en las naciones más ricas se registra una percepción ligeramente mayor de bienestar que en las más pobres; y dentro de un mismo país, los habitantes con mayor poder adquisitivo informan de niveles más altos de felicidad que el resto de la población. Sin embargo, a la vez se ha encontrado que la población de países con bajos ingresos per cápita, como Argentina, Brasil o Chile, presenta una percepción de bienestar comparable a la de los habitantes de Japón, por ejemplo (Inglehart, 1990). En el caso de México, a pesar de sus bajas rentas, también existen informes que señalan que se encuentra por delante de países europeos, como Francia o España, en niveles de felicidad (Laca, Verdugo y Guzmán, 2005). Es posible que la contradicción en los datos obtenidos se deba a la utilización del constructo "bienestar social", como equiparable a bienestar subjetivo, y entendiéndolo como un índice de la desigualdad de la distribución económica en el seno de una sociedad (Laca, Mejía y Yañez, 2010). Esta contradicción también ha dado lugar a la denominada "paradoja del crecimiento infeliz", que podría explicar la tendencia a la pérdida de bienestar en contextos individualistas de crecimiento económico sostenido, debido a las altas expectativas, la proliferación de la publicidad o la incidencia de los medios de comunicación (Lora y Chaparro, 2008). Además, en mu- chas ocasiones, el bienestar a nivel económico se ha valorado en función del juicio "objetivo" de un agente experto, desestimando la valoración que el interesado hace de su propia condición. Esta concepción no toma en cuenta la forma en que las personas experimentan estas condiciones objetivas, la importancia que le otorgan a las mismas y qué porcentaje de bienestar subjetivo experimentan a partir de ellas (Gómez et al., 2007). Fuentes y Rojas (2001) han sugerido que, más que de los ingresos económicos absolutos, el bienestar dependería de la brecha entre el ingreso real y el que se considera necesario para satisfacer las necesidades personales. De ahí las diferencias encontradas en felicidad entre países objetivamente más ricos y aquellos que tienen menos ingresos. De este modo, al intentar realizar una valoración del bienestar económico de las personas, los estudios recientes lo hacen considerando el criterio del propio individuo, más que el nivel de ingreso correspondiente a ese bajo bienestar, sin necesidad de emplear ningún indicador de activos personales para su definición (Rojas y Jiménez, 2008).

Aunque el bienestar puede depender en parte de los ingresos económicos, tal y como es experimentado por una persona, también está influido por otros factores (Fuentes y Rojas, 2001; Rojas, 2007). En términos generales, la investigación ha demostrado que las características sociodemográficas tienen una escasa influencia en el bienestar, únicamente tener pareja parece influir positivamente (Glenn y Weaver 1981; Mastekaasa 1993). Las condiciones laborales parecen ejercer cierta influencia, aunque en algunos estudios con trabajadores mexicanos se han obtenido niveles altos de bienestar global, independientemente del bienestar laboral de los trabajadores (Díaz y González, 2011). Las variables de personalidad, principalmente el neuroticismo y la extraversión, sí aparecen vinculadas a las emociones, ejerciendo un papel diferencial en los distintos indicadores del bienestar (Diener et al. 1999; Marrero y Carballeira, 2010, 2011). De acuerdo con Compton (2001) cualquier definición de felicidad o bienestar debe estar intrínsecamente ligada a los valores de cada cultura. Y los hallazgos de la investigación contribuyen a la consideración del carácter multidimensional del bienestar, que incluye tanto la satisfacción con los aspectos vitales propios de cada individuo (Palomar, 2005) como factores sobre las relaciones familiares, la autorrealización con el trabajo, las relaciones sociales, la salud, la libertad y los valores personales (Layar, 2005).

De ahí que en este estudio se hayan considerado, tanto los aspectos emocionales, como la felicidad y el afecto positivo y negativo, como la satisfacción en dominios específicos; así como la satisfacción vital a nivel global. Todo ello con el objeto de analizar las diferencias entre dos culturas: una colectivista (México) y una individualista (España). Asimismo, se pretende conocer qué componentes específicos del bienestar ejercen mayor influencia sobre el bienestar global (felicidad y satisfacción vital) de cada cultura, así como analizar si determinadas variables sociodemográficas pueden modularla. 


\section{Método}

\section{Participantes}

Se trata de un estudio de corte transversal, con una muestra de conveniencia formada por 346 estudiantes universitarios de Psicología, pertenecientes a la Universidad de San Luis Potosí en México $(N=173)$ y a la Universidad de La Laguna en España $(N=173)$. El rango de edad de la muestra mexicana se situó entre los 17 y los 34 años $(M$ : 19.29; $D T=2.45)$ y el de la muestra española entre los $17 \mathrm{y}$ los 31 años $(M: 19.82 ; D T=2.80)$. En la tabla 1, se presentan los datos sociodemográficos de las dos culturas. En ambos casos, alrededor del 78\% son mujeres. La gran mayoría de participantes están solteros, aunque un 34\% de los mexicanos y un $51 \%$ de los españoles están manteniendo una relación de pareja en el momento de la evaluación. Se trata de una muestra de estudiantes, aunque un porcentaje muy limitado (3.5\% en ambos casos), está activo laboralmente. Entre los mexicanos, un $32 \%$ consideraba que el dinero del que disponía, le permitía cubrir sus necesidades, mientras que el porcentaje fue más alto en el caso de la muestra española $(52 \%)$.

Tabla 1. Datos de las principales variables sociodemográficas en cada cultura.

\begin{tabular}{|c|c|c|c|c|}
\hline & \multicolumn{2}{|c|}{$\begin{array}{c}\text { Cultura } \\
\text { Colectivista } \\
\text { México } \\
(N=173)\end{array}$} & \multicolumn{2}{|c|}{$\begin{array}{c}\text { Cultura } \\
\text { Individualista } \\
\text { España } \\
(N=173)\end{array}$} \\
\hline & $\mathrm{N}$ & $\%$ & $\mathrm{~N}$ & $\%$ \\
\hline \multicolumn{5}{|l|}{ Sexo: } \\
\hline - Mujeres & 136 & $78.6 \%$ & 135 & $78.0 \%$ \\
\hline - Hombres & 37 & $21.4 \%$ & 38 & $22.0 \%$ \\
\hline \multicolumn{5}{|l|}{ Estado civil: } \\
\hline - Solteros & 166 & $96.0 \%$ & 162 & $93.6 \%$ \\
\hline - Casados & 3 & $1.7 \%$ & 1 & $.6 \%$ \\
\hline - Conviven en pareja & 1 & $.6 \%$ & 10 & $5.8 \%$ \\
\hline - Sin dato & 3 & $1.7 \%$ & - & - \\
\hline Tienen pareja & 59 & $34.1 \%$ & 89 & $51.4 \%$ \\
\hline Activos laboralmente & 6 & $3.5 \%$ & 6 & $3.5 \%$ \\
\hline Necesidades económicas cubiertas & 56 & $32.4 \%$ & 90 & $52.0 \%$ \\
\hline
\end{tabular}

\section{Instrumentos}

Escala de Satisfacción con la vida -SWLS- (Diener, Emmons, Larsen, y Griffin, 1985). Evalúa el juicio cognitivo sobre la satisfacción con la propia vida a nivel global a través de 5 ítems, como por ejemplo: Hasta el momento, he conseguido las cosas importantes que deseo en la vida, que se responden a través de una escala tipo Likert desde 1 (nada satisfecho) hasta 7 (muy satisfecho). La consistencia interna de esta escala es de .77 para la muestra de estudiantes mexicanos y de .83 para los españoles.

Cuestionario de Felicidad (Lyubomirsky y Lepper, 1999). Está formado por cuatro ítems referidos a la percepción del individuo sobre su felicidad en la vida, como por ejemplo: Algunas personas son muy felices en general. Disfrutan de la vida in- dependientemente de lo que suceda, sacan el máximo provecho de todo ¿Hasta qué punto le describe esta caracterización? Incluye siete opciones de respuesta, desde nada feliz hasta muy feliz. La consistencia interna de esta escala es de .77 en la muestra mexicana y de .79 en la española.

Escalas de Afecto Positivo y Negativo -PANAS- (Watson, Clark y Tellegen, 1988). Evalúa dos dimensiones: afecto positivo, que recoge la tendencia a experimentar emociones positivas, de energía y afiliación, a través de diez calificativos, como: motivado o entusiasta; y afecto negativo, que expresa sensibilidad temperamental a estímulos negativos y que recoge diez adjetivos como: nervioso o culpable. Todos ellos están referidos al momento de cumplimentar la escala, y se valoran en una escala de 0 (ausencia de la emoción) hasta 7 (presencia frecuente de la emoción). El alfa de Cronbach fue de .80 para las emociones positivas y de .83 para las negativas en la muestra de México; y en la de España, .74 para el afecto positivo y .79 para el negativo.

Satisfacción en áreas de dominio especifico. Las áreas de satisfacción evaluadas se incluyen en la entrevista (donde también se recogen diferentes variables sociodemográficas) a través de cuatro preguntas referidas a la satisfacción en los siguientes dominios vitales: satisfacción en el área sentimental, con los estudios, con la salud y con el ocio, todas ellas, medidas con una escala tipo Likert con seis opciones de respuesta (desde nada satisfecho hasta totalmente satisfecho).

\section{Procedimiento}

Uno de los miembros del equipo de investigación, que trabaja en la Universidad de San Luis Potosí, propuso a los estudiantes de primer curso de Psicología que cumplimentaran los cuestionarios en línea. En el mismo momento, en la Universidad de La Laguna, estudiantes de Psicología cumplimentaban la batería de pruebas. En ella se especificaba el carácter voluntario, el anonimato y la confidencialidad de la información recogida. El análisis de los datos se llevó a cabo con el programa estadístico SPSS (versión 18).

Se llevaron a cabo análisis chi cuadrado para conocer la homogeneidad de la muestra en las variables: sexo, edad, tener pareja y tener las necesidades económicas cubiertas. Se realizaron análisis de correlación parcial entre las variables de bienestar subjetivo: satisfacción vital, felicidad, emociones positivas, emociones negativas, satisfacción en el área sentimental, satisfacción con los estudios, con la salud y con el ocio, en cada uno de los dos grupos culturales. Se controlaron aquellas variables que no habían mostrado homogeneidad entre las muestras en los análisis previos. Se llevó a cabo un ANOVA en cada cultura para comprobar si tener la economía cubierta, ejercía influencia sobre el bienestar. Se aplicó un MANCOVA controlando las variables edad y tener pareja, que no habían mostrado homogeneidad entre los grupos, para conocer las diferencias entre ambas culturas en las medidas de bienestar subjetivo. Se tomó como variable independiente la cultura: colectivista $v s$. individualista y como variables dependientes los cuatro indicadores de bienestar 
subjetivo y los cuatro índices de satisfacción en dominios específicos. Finalmente, se llevaron a cabo cuatro análisis de Regresión Múltiple, dos en cada cultura (colectivista e individualista), para las variables criterio: satisfacción vital y felicidad. Las variables independientes fueron: sexo, edad, tener pareja y tener la economía cubierta; y los cuatro índices de satisfacción en dominios específicos: satisfacción sentimental, con los estudios, con la salud y con el ocio.

\section{Resultados}

En primer lugar, se han llevado a cabo análisis chi cuadrado para analizar si las variables: sexo, edad, tener pareja, y percepción de tener la economía cubierta, eran homogéneas en función de la cultura de procedencia. Los resultados mostra- ron que los grupos fueron homogéneos en sexo $\left(\chi^{2}(1)=\right.$ $.017 ; p=.896)$ y en la percepción de tener la economía cubierta $\left(\chi^{2}(1)=4.004 ; p=.065\right)$, existiendo diferencias en edad $\left(\chi^{2}(13)=39.011 ; p<.000\right)$ y en tener pareja $\left(\chi^{2}(1)=\right.$ 10.627; $p<.001)$.

A continuación, con el fin de conocer las asociaciones más destacadas entre las variables de bienestar subjetivo en cada muestra, se realizaron análisis de correlación parcial, controlando las variables sociodemográficas que no habían mostrado homogeneidad en ambas muestras: edad y tener pareja. Dichos análisis han mostrado índices de relación semejantes en ambas culturas entre la gran mayoría de variables de bienestar, aunque algunas de las correlaciones muestran patrones diferentes (ver tabla 2).

Tabla 2. Correlaciones parciales (controlando edad y tener pareja) entre las variables de bienestar subjetivo en ambas muestras de estudiantes

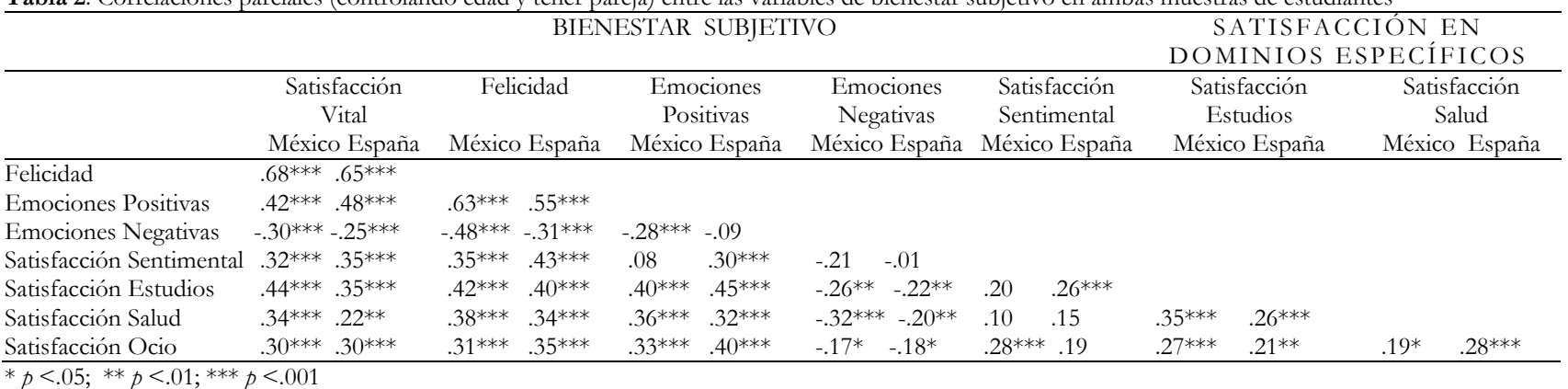

$* p<.05 ; * * p<.01 ; * * * p<.001$

Se observa una relación positiva y de moderada magnitud entre felicidad y satisfacción vital ( $r=.68$ en estudiantes mexicanos y $r=.65$ en los españoles), pero el porcentaje de varianza compartida no supera el $46 \%$, por lo que podemos señalar que se trata de constructos diferentes. Del mismo modo, las emociones positivas, aunque se han entendido como un componente del bienestar, concretamente de la felicidad, y siendo tratadas como un mismo concepto, únicamente comparten con esta variable el $30 \%$ de la varianza en la muestra española y el $40 \%$ en la mexicana. También las emociones positivas se presentan como entidades independientes de la satisfacción vital, cuyas relaciones son sensiblemente más bajas ( $r=.42$ en estudiantes mexicanos y $r=$ .48 en los españoles) que las que mantienen con la felicidad. Como es esperable, las emociones negativas se asocian inversamente con todas las medidas de bienestar. Tal y como ocurre con las positivas, aquellas mantienen correlaciones de mayor magnitud con felicidad $(r=-.48$ en estudiantes mexicanos y $r=-.31$ en los españoles) que con satisfacción vital $(r=-.30$ en estudiantes mexicanos y $r=-.25$ en los españoles). Las emociones positivas y negativas se relacionan entre sí en los estudiantes mexicanos $(r=-.28)$ no existiendo relación en la muestra de estudiantes españoles $(r=-.09)$.

Las asociaciones de los cuatro dominios de satisfacción específicos con satisfacción vital y felicidad oscilan entre .22 y .44, dándose ciertas diferencias en los índices de correlación en ambas muestras. Así, en la muestra mexicana, las re- laciones de mayor magnitud se dan entre la satisfacción con los estudios y la satisfacción vital $(r=.44)$ y también con la felicidad $(r=.42)$. Mientras que en la española, es la satisfacción sentimental la que mantiene relaciones más intensas con felicidad $(r=.43)$ y con la satisfacción vital; aunque en este último caso, la satisfacción con los estudios también alcanza el mismo nivel de asociación $(r=.35)$. Las relaciones que la satisfacción sentimental mantiene con el afecto también difieren en ambas culturas. Entre los estudiantes españoles, las emociones positivas se asocian a la satisfacción en la relación de pareja $(r=.30)$, pero no las negativas. En la muestra mexicana ocurre lo contrario, es el afecto negativo el que se asocia con la satisfacción sentimental $(r=-.21)$. Esta también se relaciona con la satisfacción con la salud para los españoles $(r=.15)$ y con la satisfacción con el ocio en ambas culturas, pero fundamentalmente en la mexicana $(r=$ $.28)$, frente a la española $(r=.19)$.

También se procedió a realizar un ANOVA en cada grupo cultural para comprobar si el hecho de tener la economía cubierta, podía incidir en el bienestar. Los resultados mostraron que el nivel de bienestar de la muestra mexicana es independiente de tener las necesidades económicas cubiertas, mientras que en la muestra española, aquellos que tienen sus necesidades económicas cubiertas, informaron de mayor felicidad $(F(1,171)=6.254 ; p=.013)$.

Con el fin de conocer si se daban diferencias en los distintos indicadores de bienestar, según la cultura a la que per- 
tenecía la muestra, se realizó un MANCOVA tomando como variable independiente la cultura de procedencia de la muestra (colectivista $v$ s. individualista) y como variables dependientes los ocho indicadores de bienestar, controlando la edad y el hecho de tener pareja. Existe un efecto multivariado $(\lambda=0.39 ; F=59.98, p=0.000)$. Los resultados indican que los estudiantes mexicanos presentan mayor bienestar subjetivo que los españoles, mostrando mayor satisfacción vital $[F(1,317)=64.63 ; p<.000 ; \eta 2=.17] ;$ mayor felicidad $[F(1,317)=58.41 ; p<.000 ; \eta 2=.15]$; más emociones positivas $[F(1,317)=87.76 ; p<.000 ; \eta 2=.22]$; y menos emociones negativas $[F(1,317)=17.00 ; p<.000 ; \eta 2=.05]$. También la satisfacción en áreas específicas fue más alta en la muestra mexicana, informando de mayor satisfacción en el área sentimental $[F(1,317)=17.78 ; p<.01 ; \eta 2=.05]$, con los estudios $[F(1,317)=327.89 ; p<.000 ; \eta 2=.51]$, con la salud $[F(1,317)=106.57 ; p<.000 ; \eta 2=.25]$ y con el ocio $[F(1,317)=250.52 ; p<.000 ; \eta 2=.44]$. Teniendo en cuenta los índices del tamaño del efecto de los análisis, que oscila- ron entre 0.05 y 0.44 , obtenidos a través de Eta cuadrado, las diferencias halladas entre ambas culturas deben ser tomadas en consideración.

A partir de los resultados obtenidos en el MANCOVA, que muestran la superioridad de la muestra mexicana en la mayoría de los índices de bienestar, se pretendía conocer qué variables influían en mayor medida en las dos variables globales de bienestar subjetivo: satisfacción vital y felicidad. En ambas muestras de estudiantes se realizaron análisis de regresión múltiple, método hacia delante. Como variables independientes se tomaron: el sexo, la edad, el hecho de tener pareja, tener la economía cubierta, además de los cuatro índices de satisfacción en dominios específicos: satisfacción sentimental, con los estudios, con la salud y con el ocio.

Los resultados de los análisis de regresión múltiple realizados sobre la satisfacción vital y la felicidad en ambas muestras se presentan en la tabla 3, e incluye las variables que entraron en el último paso de cada regresión.

Tabla 3. Análisis de regresión múltiple de variables sociodemográficas y satisfacción en dominios específicos sobre satisfacción vital y felicidad en ambas culturas.

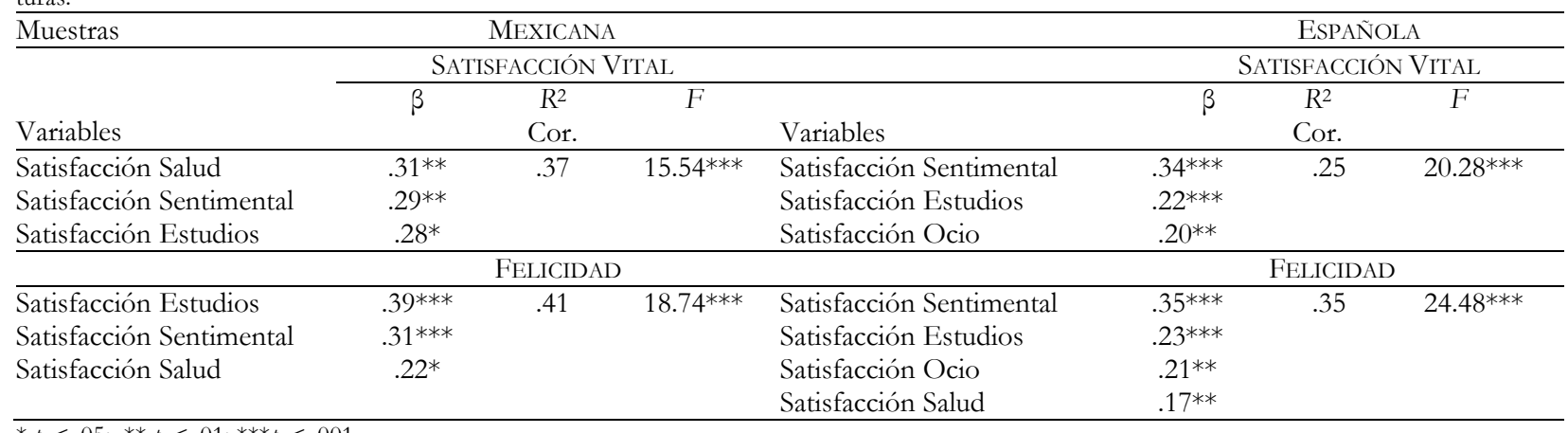

$* p<.05 ; * * p<.01 ; * * p<.001$

Las variables que predijeron la satisfacción vital de los estudiantes mexicanos fueron: satisfacción con la salud $(\beta=.31 ; p=.008)$, satisfacción sentimental $(\beta=.29 ; p=.003)$ y satisfacción con los estudios $(\beta=.28 ; p=.014)$. Todas ellas explicaron el $37 \%$ de la varianza de dicha variable $[F(3,72)=15.54 ; p<.000]$. Entre los estudiantes españoles, la satisfacción sentimental $(\beta=.34 ; p<.000)$, la satisfacción con los estudios $(\beta=.22 ; p<.001)$, y la satisfacción con el ocio $(\beta=.20 ; p=.003)$, explicaron el $25 \%$ de la varianza de la satisfacción vital $[F(3,169)=20.28 ; p<.000]$.

En la muestra mexicana, la satisfacción con los estudios $(\beta=.39 ; p<.001)$, la satisfacción sentimental $(\beta=.31 ; p<$ $.001)$ y la satisfacción con la salud $(\beta=.22 ; p=.042)$ explicaron un $41 \%$ de la varianza de la felicidad $[F(3,72)=18.74 ; p<$ $.000]$. Por lo que se refiere a los estudiantes españoles, la satisfacción sentimental $(\beta=.35 ; p<.000)$, con los estudios $(\beta=$ $.23 ; p<.000)$, con el ocio $(\beta=.21 ; p<.001)$ y con la salud $(\beta=.17 ; p=.010)$ predijeron el $35 \%$ de la varianza de la felicidad $[F(4,168)=24.48 ; p<.000]$.
Ninguno de los índices sociodemográficos considerados, pudieron explicar la felicidad ni la satisfacción vital de ambas muestras.

\section{Discusión}

El principal objetivo de este estudio consistió en analizar las diferencias en el bienestar subjetivo de estudiantes provenientes de dos culturas: una colectivista y una individualista. Los resultados han mostrado que la cultura colectivista presenta mayor bienestar que la individualista, tanto en los indicadores generales: satisfacción vital, felicidad, afecto positivo y negativo, como en los específicos relacionados con distintos dominios vitales. Hasta el momento, una parte de la investigación apuntaba al mayor bienestar de las sociedades individualistas aunque, tal y como se ha recogido aquí, los hallazgos habían sido contradictorios.

Los resultados de este estudio corroboran algunos trabajos previos que señalan la universalidad del constructo bienestar. Así, en ambas muestras el patrón de relación entre los indicadores de bienestar fue similar, estando las medidas 
globales de dicho constructo más relacionadas entre sí que las específicas. Hay que tener en cuenta que las distintas medidas aportan una parte de varianza única al nivel de bienestar global de los individuos, revelando cierta independencia entre ellas y, tal y como defienden algunos autores (Pavot y Diener, 1993), justificando su uso complementario en los estudios sobre el tema. En el caso de las emociones positivas y negativas se observa una independencia entre las mismas para los estudiantes españoles, mientras que en los mexicanos aparece cierto nivel de dependencia, pareciendo de alguna manera, interferir la presencia de afecto positivo con la presencia del negativo o viceversa. La escala empleada ha sido creada y validada con población anglosajona, lo que podría influir en este resultado. Aunque para aludir a las emociones, se han utilizado los términos más utilizados y adecuados para una mejor comprensión en ambas culturas, entendemos que la idiosincrasia de la misma puede contribuir a la forma en que se experimentan, manifiestan y verbalizan las emociones. Indudablemente habría que seguir profundizando en este tema, no solo considerando la ausencia o presencia de cada emoción en el momento en que los participantes cumplimentan las pruebas, sino analizando de forma más exhaustiva su frecuencia e intensidad, lo que podría contribuir a complementar la evaluación del afecto, tal y como sugieren autores de prestigio en este tema (Diener, Larsen, Levine, y Emmons, 1985).

La satisfacción sentimental parece jugar un papel importante en el bienestar de la muestra española, pero no resulta tan relevante para los mexicanos, quizás porque una gran parte de ellos no está manteniendo una relación de pareja en el momento de la evaluación.

Según Lang y Heckhausen (2001) las personas serán más felices y estarán más satisfechas en la medida en que puedan realizar con éxito sus tareas diarias y obtener sus metas personales. Según esto, sería esperable que aquellos individuos pertenecientes a una cultura individualista, donde abundan valores como el logro, tenderían a informar de mayor bienestar. Sin embargo, los resultados obtenidos en el presente estudio van en la línea opuesta. Es posible que, en este caso, prime otro tipo de variables que hagan incrementar dichos indicadores de bienestar. De acuerdo con Compton (2001), cualquier definición de felicidad o bienestar está intrínsecamente ligada a los valores de cada cultura, por lo que, indudablemente, no es solo cuestión de analizar de forma cuantitativa hasta qué punto se siente que la vida "está llena" en determinados aspectos, como la satisfacción en las áreas específicas, sino posiblemente ir más allá, e intentar descubrir el significado que tiene para cada persona ese hecho en función de la procedencia cultural. Schmuck y Sheldon (2001) sugieren que en la medida en que las personas se preocupen por metas "más allá de sí mismas", menos centradas en gratificaciones materiales inmediatas, y más en relaciones interpersonales estrechas que permitan tener una visión más integrada de sí mismos, tenderán a percibir mayor bienestar y felicidad. Y esto parece darse, en mayor medida, en las sociedades colectivistas. Nuestros resultados parecen seguir esa línea ya que se muestran poco modulados por la percepción subjetiva sobre la economía personal, pues la mitad de los españoles y casi el setenta por ciento de los mexicanos, no percibe tener sus necesidades económicas cubiertas y, curiosamente, estos últimos informan de mayor bienestar. Parece revelarse de ello que disponer de una economía saneada no garantiza el bienestar, lo que además está en consonancia con los hallazgos de otros estudios en los que bienestar personal y nivel económico no parecen ir asociados (Inglehart, 1990).

Por otra parte, México es un país emergente en el que los individuos pueden percibir que sus condiciones de vida están mejorando, lo que parece tener un incremento inmediato en la satisfacción con la vida de estos países menos ricos, pero no repercutir tan positivamente en los países que ya tienen gran parte de sus necesidades cubiertas (Matijasevic, Ramírez y Villada, 2010). Esto va en la línea de lo argumentado por Lora y Chaparro (2008) sobre la estabilización a la baja del bienestar en las sociedades individualistas tras las altas expectativas generadas y la expansión del crecimiento sostenido a través de los medios de comunicación. Si a eso unimos la actual situación económica por la que están pasando muchas sociedades desarrolladas, y más concretamente España, disponemos de explicaciones para este hallazgo. Como el que se confirma con la relación positiva entre percepción de economía saneada y felicidad en la muestra española.

Cuando se analizan los principales predictores del bienestar global, se observa el valor que otorga cada cultura a los diferentes componentes específicos del mismo. En ambos grupos culturales la satisfacción sentimental, con la salud y con los estudios explican una parte, tanto de la satisfacción vital como de la felicidad. Sin embargo el peso relativo de cada variable difiere en ambas culturas. Para los mexicanos es más importante la satisfacción con la salud, mientras que para los españoles la satisfacción con el ocio también explica parte del bienestar. Parece que la felicidad y la satisfacción vital de la muestra mexicana están más determinadas por aspectos propios de la edad de los estudiantes universitarios, como puede ser la satisfacción a nivel afectivo y también el sentirse satisfecho con los estudios, por ser ambas, áreas importantes en esta etapa vital. Además, el hecho de que la satisfacción con la salud para los mexicanos, y la satisfacción con el ocio para los españoles, sean importantes predictores del bienestar, podría ser debido a que, probablemente, la salud es una necesidad más básica que el ocio, cobrando mayor importancia en países que han estado desfavorecidos.

Algunos estudios sugieren que el balance afectivo entre emociones positivas y negativas, es un predictor más fuerte de satisfacción vital en las culturas individualistas que en las culturas colectivistas (D'Anello, 2006; Suh, Diener, Oishi, y Triandis, 1998). Esto se da cuando en las sociedades individualistas prima la búsqueda de emociones positivas y la evitación de negativas, frente a las sociedades colectivistas, en las que podrían adquirir más valor otras cuestiones. Quizás los estudios o la salud sean esas cuestiones que, con carácter 
positivo, también están ineludiblemente unidas en cualquier individuo a aspectos negativos. Es decir son más heterogéneas (cuando por ejemplo se dan problemas de salud o ante el esfuerzo que requieren los estudios, aunque a la postre genere recompensas). Frente a ellas, la satisfacción sentimental o con el ocio, pueden ser entendidas de forma más homogénea y, a priori, tener más que ver con la búsqueda de los aspectos más hedonistas del bienestar.

El estudio no está exento de ciertas limitaciones, por lo que los resultados deben ser tomados con precaución. La muestra está limitada a estudiantes de Psicología, habría que analizar otro tipo de población más heterogénea, para contrastar los resultados. Según algunos autores (D'Anello, 2006), los estudiantes universitarios, aunque pertenecientes a culturas colectivistas, están más propensos a recibir la influencia de las sociedades individualistas por sus posibilidades de acceso tanto a la cultura como a la información, lo que en la muestra de estudiantes mexicanos podría estar difuminando la influencia de pertenecer a una cultura colecti-

\section{Referencias}

Argyle, M. (1992). La Psicología de la Felicidad. Madrid: Alianza Editorial. Compton, W. (2001). The values problem in Subjective Well-being. American Psychological Association, 56, 1-84.

D'Anello, S. (2006). Efecto de las Normas y Emociones en los Juicios sobre Satisfacción con la Vida en una Muestra Venezolana. Revista Interamericana de Psicologia/Interamerican Journal of Psychology, 40, 371-376.

DeNeeve, K. M. y Cooper, H. (1998). The happy personality: A meta-analysis of 137 personality traits and subjective well-being. Psychological Bulletin, 124, 197229.

Díaz, F. y González, M. J. (2011). Subjective and Occupational Well-Being in a Sample of Mexican Workers. Social Indicators Research, 100, 273-285.

Diener, E. (2000). The science of happiness and a proposal for a national index. American Psychological Association, 55, 34-43.

Diener, E., Emmons, R. A., Larsen, R. J. y Griffin, S. (1985). The Satisfaction with Life Scale. Journal of Personality Assessment, 49, 71-75.

Diener, E., Larsen, R. J., Levine, S. y Emmons, R. (1985). Intensity and frequency: Dimensions underlying positive and negative affect. Journal of Personality and Social Psychology. 48, 1253-1265.

Diener, E., Suh, E., Lucas, R. y Smith, H. (1999). El bienestar subjetivo, tres décadas de progreso. Boletín Psicológico. 125, 271-301.

Fuentes, N. y Rojas, M. (2001). Economic Theory and Subjective Well-Being: Mexico. Social Indicators Research, 53, 289-314.

Furman, R., Negi, N. J., Iwamoto, D. K., Rowan, D., Shukraft, A., y Gragg, J. (2009). Social work practice with latinos: Key issues for social workers. Social Work, 54, 167-174.

Glenn, N. D., y Weaver, C. N. (1981). The contribution of marital happiness to global happiness. Journal of Marriage and the Family, 43, 161-168.

Gómez, V., Villegas, C., Barrera, F. y Cruz, J. E. (2007). Factores predictores de Bienestar Subjetivo en una muestra colombiana. Revista Latinoamericana de Psicología, 39, 311-325.

Hofstede, G. (1999). Culturas y organizaciones. Madrid: Alianza Editorial.

Inglehart, R. (1990). Culture shift in advanced industrial society. Princeton, NJ: Princeton University Press.

Laca, F. A., Mejía, J. C. y Yáñez, C. (2010). Identidad mexicana e interés político: Predictores de bienestar social y anomia. Acta Universitaria, 20, 40-49.

Laca, F. A., Verdugo, J. y Guzmán, J. (2005). Satisfacción con la vida de algunos colectivos mexicanos: una discusión sobre la psicología del bienestar subjetivo. Enseñanza e Investigación en Psicología 10, 325-336.

Lang, F. y Heckhausen, J. (2001). Perceived control over development and subjective well-being differential benefits across adulthood. American Psychological Association, 81, 509-523.

Layar, R. (2005). La felicidad: Lecciones de una nueva ciencia. Madrid: Taurus.

Lora, E., y Chaparro, J. C. (2008). La conflictiva relación entre ingreso y la satisfacción. En E. Lora (Coord.), Calidad de vida. Más allá de los hechos (pp. 1-50). vista. Además, ciertas características psicológicas, como las variables de personalidad, las estrategias de afrontamiento o la percepción de apoyo social, que no se han tomado en consideración aquí, podrían ser relevantes para identificar otros factores que subyacen al bienestar de culturas diferenciadas. De hecho, algunos autores (Gómez et al., 2007) han encontrado que ciertos factores, como la autoeficacia o el control personal, están relacionados con la satisfacción con los estudios o con la vida sentimental.

Los hallazgos de la presente investigación parecen indicar que las sociedades emergentes, como es el caso de México, están incrementando su bienestar personal en detrimento de las denominadas hasta hace poco sociedades "avanzadas". Consideramos que las demandas y el afán de logro de países con valores básicamente individualistas, como España que, además, en la actualidad sufre una grave crisis económi$\mathrm{ca}$, han encorsetado a sus ciudadanos para conseguir metas casi inalcanzables, lo que puede estar repercutiendo negativamente en su bienestar.
Washington: Banco Interamericano de Desarrollo y Fondo de Cultura Económica.

Lyubomirsky, S. y Lepper, H. S. (1999). A measure of subjective happiness: Preliminary reliability and construct validation. Social Indicators Research, 46, 137155.

Marrero, R. J. y Carballeira, M. (2010). El papel del optimismo y del apoyo social en el bienestar subjetivo. Salud Mental, 33, 39-46.

Marrero, R. J. y Carballeira, M. (2011). Well-being and personality: Facet-level analyses. Personality and Individual Differences, 50, 206-211.

Mastekaasa, A. (1993). Marital status and subjective well-being: A changing relationship? Social Indicators Research, 29, 249-276.

Matijasevic, M. T., Ramírez, M. y Villada, C. (2010). Bienestar subjetivo: Una revisión crítica de sus resultados, alcances y limitaciones. Regiones, 5, 5-39.

Palomar, L. J. (2005). Estructura del bienestar subjetivo: construcción de una escala multidimensional. En E. L. Garduño, A. B. Salinas, y H. M. Rojas. Calidad de Vida y Bienestar Subjetivo en México (pp. 113-142). México D.F.: Plaza y Valdez S.A.

Pavot, W. y Diener, E. (1993). Review of the Satisfaction with life scale. Psychological Assessment, 5, 164-172.

Rice, T., y Steele, B. (2004). Subjective well-being and culture across time and space. Journal of Cross-Cultural Psychology, 35, 633-647.

Rojas, M. (2007). Heterogeneity in the Relationship between Income and Happiness: A Conceptual Referent Theory Explanation. Journal of Economic Psycho$\log y, 28,1-14$.

Rojas, M. y Jiménez, E. (2008).Pobreza subjetiva en México: el papel de las normas de evaluación del ingreso. Perfiles Latinoamericanos, 32, 11-33.

Schimmack, U., Radhakrishnan, P., Oishi, S., y Ahadi, S. (2002). Culture, personality, and subjective well-being. Integrating process models of life satisfaction. Journal of Personality and Social Psychology, 82, 582-593.

Schmuck, P. y Sheldon, K. M (2001). Life goals and well-being. Towards a positive psychology of human striving. Seattle, WA: Hogrefe \& Huber.

Suh, E., Diener, E., Oishi, S., y Triandis, H. C. (1998). The shifting basis of life satisfaction judgments across cultures: Emotions versus Norms. Journal of Personality and Social Psychology, 74, 482-493.

Triandis, H. C. (1980). Handbook of cross cultural psychology. Vol.2. U.S.A. Allyn and Bacon.

Triandis, H. C. (1990). Multimethod probes of individualism and collectivism. Journal of personality and Social Psychology, 59, 1006-1020.

Triandis, H. C. (1995). Individualism and collectivism. Boulder, USA: Westview Press. Triandis, H. C. (2005). Issues in individualism and collectivism research. En R.M. Sorrentino, D. Cohen, J. M. Olson, y M. P. Zanna (Eds.), Cultural and Social Behavior: The Ontario Symposium, (pp. 207-224). Mahawah, NJ: Lawrence Erlbaum Associates.

Veenhoven, R. (1984). Conditions of Happiness. Dordrecht: Reidel. 
Veenhoven, R. (2007). Trend Average Happiness in Nations 1946 - 2006: How much people like the life they live. World Database of Happiness. Trend Report, 1. World Database of Happiness. Recuperado de: http://worlddatabaseofhappiness.eur.nl/hap_nat/findingreports/TrendRep ort2007-1.pdf

Vera, J. A., Laborín, J. F., Córdova, M. A. y Parra, E. M. (2007). Bienestar Subjetivo: comparación en dos contextos culturales. Psicología para América Latina.
Revista de la Unión Latinoamericana de Entidades de Psicología. Recuperado de http://psicolatina.org/11/(5 de 7)25/10/2007 11:28:24

Watson, D., Clark, L. A. y Tellegen, A. (1988). Development and validation of brief measures of positive and negative affect: The PANAS Scales. Journal of Personality and Social Psychology, 54, 6, 1063-1070.

(Articulo recibido: 22-1-2013; revisión recibida: 8-6-2013; aceptado: 5-8-2013) 